\title{
Utilización de Modelos y Teorías de Enfermería de Escuelas del Ser Humano Unitario y Caring en Servicios del Hospital María Auxiliadora
}

\author{
Using Models and Theories of Nursing Unit of the Human Being and Caring Schools in the \\ HMA Services \\ Nancy Huamán Salazar1, Angélica Miranda Alvarado², Justina Violeta Vásquez Ramírez², Berna Luzgarda Gonzales

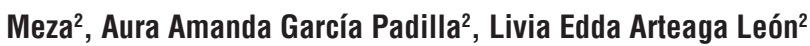

\begin{abstract}
RESUMEN
Los modelos y teorías, en la práctica de la enfermería, orientan el quehacer del enfermero que va más allá de la técnica y permiten guiar las actividades, mejorando la gestión de los cuidados de enfermería en los servicios de salud. Objetivo: Determinar la utilización de modelos y teorías de enfermería de las Escuelas del Ser Humano Unitario y el Caring, por las enfermeras del Hospital María Auxiliadora. Material y métodos: Estudio de nivel aplicativo, descriptivo y de corte transversal, tuvo una muestra de 137 enfermeras, se usó la técnica de la encuesta y el cuestionario como instrumento. Resultados: El profesional de enfermería utiliza los modelos y teorías de enfermería en la conceptualización cuidado en un $8 \%$, persona en $34 \%$, salud, un $19 \%$ y entorno en un $19 \%$. De la Escuela del Ser Humano Unitario utilizan un 12\% cuidado, el $23 \%$ persona, un $8 \%$ salud, el $13 \%$ entorno y de la Escuela del Caring, el 4\% utilizan el cuidado, un 55\% persona, el 69\% salud y 25\% entorno. Conclusiones: El paradigma de transformación ubica a las escuelas del ser humano y el caring, las concepciones de estas teorías en su dimensión persona, salud, entorno son utilizadas en muy bajo porcentaje por el profesional de enfermería.
\end{abstract}

Palabras clave: Modelos, teorías, enfermería, ser humano unitario, caring.

\begin{abstract}
Models and theories in nursing practice nurse oriented task that goes beyond the art and help guide activities to improve the management of nursing care in health services. Objective: To determine the use of nursing models and theories of Human Being School and Caring Unit for nurses Maria Auxiliadora Hospital. Methods: Study of application, descriptive and cross-sectional level, took a sample of 137 nurses, technical survey and questionnaire instrument was used. Results: The nurse uses the models and theories in conceptualizing nursing care by $8 \%, 34 \%$ person, health, environment $19 \%$ and $19 \%$. School of Human Unit use care $12 \%, 23 \%$ person, $8 \%$ health, $13 \%$ environment and Caring School, 4\% use care, a person 55\% 69\% Health and 25\% environment. Conclusions: The paradigm of transformation located schools and caring man, conceptions of these theories in his person, health, environment dimension are used in very low percentage for the nurse.
\end{abstract}

Keywords: Models, theories, Nursing, People Unit, Caring.

\footnotetext{
${ }^{1}$ Docente de la EP de Enfermería, Facultad de Medicina, Universidad Nacional Mavor de San Marcos. Lima, Perú.

${ }^{2}$ Enfermeras supervisoras del Departamento de Enfermería, Hospital María Auxiliadora. Lima, Perú.
} 


\section{INTRODUCCIÓN}

Los modelos y teorías, en la práctica de la enfermería, son muy importantes porque orientan el quehacer enfermero que va más allá de la técnica (Duran de Villalobos 2002 \& Marriner, 2003) y permiten guiar las actividades, mejorando la gestión de los cuidados de enfermería en los servicios de salud. Existen modelos y teorías descritas ampliamente que evidencian las diferencias históricas en el pensamiento enfermero, siendo la categorización, la integración y la transformación los paradigmas relevantes (Kerouác 1996).

Los metaparadigmas son los conceptos globales del fenómeno de interés de la disciplina de enfermería; persona, salud, entorno y enfermería, los cuales han sido conceptualizados de manera diferente por distintas enfermeras, para dar lugar a la generación de modelos conceptuales. (Cisneros, 1996).

Un estudio a nivel nacional, realizado en Lima Este, sobre factores influyentes en la aplicación de modelos y teorías de enfermería, para el cuidado del paciente, identificó que los profesionales de enfermería aplican modelos y teorías mayormente por factores personales en $50 \%$, lo consideran importante por la ayuda en su labor el $63.6 \%$, recibieron enseñanza en el pregrado y están motivados en su aplicación el $59.1 \%$. Entre los factores institucionales presentes como razones del bajo porcentaje, en su aplicación, comprenden: menos del $25 \%$, por la elevada demanda y tienen facilidades para asistir a cursos que les ayuden, en este campo, el 18.2\% (Armas, 2010).

Otro estudio internacional, realizado en España sobre Aplicabilidad de Modelos y Teorías de Enfermería en la realidad asistencial, evidenció una gran variabilidad de posicionamiento teórico de los profesionales de enfermería, el metaparadigma enfermero no surge de una única teoría, un dato importante es que solo $23.5 \%$ posee formación continuada en el tema. (Santos, López \&Varez, 2007).

A pesar que el servicio fundamental de enfermería es el cuidado, este no ha cambiado, pues sigue siendo el quehacer esencial y la expresión más directa. Los ambientes en que se desarrolla y se desarrollará en un futuro próximo se ligan, de un lado, a complejas tecnologías y, de otro, nuevamente hacia la Atención Primaria en Salud (Vásquez 2007). Sin embargo, amerita que esa tendencia se realice dentro de un marco holístico e integral, y que el cuidado de enfermería se exprese de manera humanística y científica.
Los diferentes abordajes conceptuales en enfermería nos conducen de manera sustancial, no solo a ver al ser humano en su totalidad, sino que interactúa en un ambiente social y natural, y con un nuevo lenguaje que entiende sistemas más complejos e integradores en una comunidad global.

Los modelos conceptuales y las teorías están elaboradas por conceptos que describen imágenes mentales de los fenómenos, no se limitan a un grupo, situación e individuo en particular, sino son generales, estos se relacionan para explicar distintos fenómenos de interés para la disciplina, como lo hicieron: Orem sobre el déficit de autocuidado; Roy, adaptación y estímulo; Henderson, necesidades básicas; Pender, conducta promotora de salud y Neuman, estresores, entre otros (Fawcett, 1984).

\section{MATERIAL Y MÉTODOS}

Es un estudio de nivel aplicativo, descriptivo y de corte transversal.

El área de estudio corresponde a los Servicios de Enfermería del Hospital María Auxiliadora, ubicado en la Av. Miguel Iglesias No 968 - San Juan de Miraflores, su área de jurisdicción comprende los distritos de San de Miraflores, Villa María del Triunfo, Villa El Salvador y otros distritos como Surco, Barranco y Chorrillos.

El Departamento de Enfermería es una unidad orgánica, encargada de ejecutar las acciones y procedimientos de enfermería que aseguren la atención integral e individualizada del paciente de acuerdo a su grado de dependencia, diagnóstico y tratamiento médico, proyectando sus acciones a la familia y a la comunidad. Se organiza estructuralmente en cinco servicios asistenciales, cada uno de ellos con objetivos funcionales específicos orientados complementariamente al logro de los objetivos funcionales del departamento. Estos son: Servicio de Enfermería: Consulta Externa, Emergencia y Cuidados Críticos, Centro Quirúrgico y Central de Esterilización, Servicio de Enfermería en Hospitalización, Atención Preventivo Promocional.

La población estuvo conformada por todas las enfermeras que laboran en los servicios de enfermería del hospital, la muestra obtenida fue por estratos en cada área o servicio de enfermería y se obtuvo una muestra de 137 enfermeras. Quienes cumplieron con los criterios establecidos: enfermeras que laboran en el hospital, con un mínimo de 1 año y que voluntariamente aceptaron participar. 
La técnica de recolección de datos fue la encuesta $\mathrm{y}$ el instrumento el cuestionario, cuya validez se obtuvo mediante 7 jueces expertos y la aplicación de la prueba binomial con un valor $\mathrm{p}$, menor 0,05 , siendo la concordancia significativa $(\mathrm{p}=0,008)$.

En el procedimiento de recolección de datos se tuvo en cuenta el permiso de la institución, las coordinaciones con el personal de salud del área (Departamento de Enfermería y los Servicios de Enfermería en las diferentes áreas), así como con las unidades competentes (Dirección General, Oficina de Apoyo a la Docencia e Investigación).

La aplicación del cuestionario se realizó individualmente a cada enfermera saliente, en turno diurno y nocturno. Terminada la recolección de datos se procedió al procesamiento, mediante la aplicación del programa estadístico SPSS Versión.17 y Excel 2007. La presentación de los datos fue a través de tablas de doble entrada y el análisis e interpretación a la luz de la literatura.

\section{RESULTADOS}

Del total de la muestra seleccionada (137), según criterios de inclusión y exclusión, se encontró que el 64\% fueron del área de Hospitalización, 33\% del área Unidades Críticas, 4.3\% del área de Centro Quirúrgico, $5.8 \%$ del área Preventivo-Promocional y 29\% del área de la Consulta Externa.

El $94 \%$ fueron del sexo femenino. En relación a la edad, el $31.6 \%$ comprende entre las edades de 36 a 45 años, $31.6 \%$ entre 46 a 55 años, el $27 \%$ entre 25 a 35 años, y el $9.6 \%$ entre 56 a 65 años.

El tiempo de servicio, en el Hospital, se evidenció que el $30.8 \%$ tenía menos de 5 años; el $25.7 \%$ entre 21 a 25 años; el $13.24 \%$, entre 11 a 15 años; el $12.5 \%$ entre 16 a 20 años; el $11 \%$ entre 6 a 10 años y el $6.6 \%$, entre 26 a 30 años.

El tiempo laborando, en el servicio actual, comprende: el $23.5 \%$ menos de un año; $21.32 \%$ de 2 a 5 años; el $19.1 \%$ de 6 a 10 años; el $13.2 \% 11$ a 15 años, el $19.12 \%$ (26) de 21 a 30 años y un $3.6 \%$ tenía de 16 a 20 años de servicios.

Tabla 1

Distribución porcentual de la utilización de los Modelos y Teorías de enfermería de la Escuela del Ser Humano y el Caring por las enfermeras del Hospital María Auxiliadora, Lima 2011.

\begin{tabular}{cllll}
\hline \multirow{2}{*}{ Dimensiones } & \multicolumn{3}{l}{ Modelos y Teorías de Enfermería } \\
\cline { 2 - 5 } & $\mathrm{N}$ & Utilizan & $\mathrm{N}$ & $\%$ \\
\hline Cuidado & 11 & 8 & 126 & 92 \\
Persona & 47 & 34 & 90 & 66 \\
Salud & 53 & 39 & 83 & 61 \\
Entorno & 26 & 19 & 111 & 81 \\
\hline
\end{tabular}

En la tabla 1, se presenta la distribución porcentual de la utilización de los Modelos y Teorías de Enfermería de la Escuela del Ser Humano y del Caring por las enfermeras, de un total de 137 enfermeras, que representa el $100 \%$, se encontró que utilizan el $8 \%$ en cuidado, $34 \%$ en persona, $39 \%$ en salud y $19 \%$ entorno; sin embargo, no utilizan los modelos y teorías el $92 \%$ en cuidado, $66 \%$ en persona, $61 \%$ en salud y $81 \%$ en entorno. 
Nancy Huamán Salazar, Angélica Miranda Alvarado, Justina Violeta Vásquez Ramírez, Berna Luzgarda Gonzales Meza, Aura Amanda García Padilla, Livia Edda Arteaga León

Tabla 2

Distribución porcentual de la utilización de los Modelos y Teorías de Enfermería de la Escuela del Ser Humano por las enfermeras del Hospital María Auxiliadora. Lima-2011.

\begin{tabular}{|c|c|c|c|c|c|c|}
\hline \multirow{3}{*}{ Dimensiones } & \multicolumn{6}{|c|}{ Escuela del Ser Humano Unitario } \\
\hline & \multicolumn{2}{|c|}{ Utilizan } & \multicolumn{2}{|c|}{ No utilizan } & \multirow[t]{2}{*}{ Total } & \multirow[t]{2}{*}{$\%$} \\
\hline & $\mathrm{N}$ & $\%$ & $\mathrm{~N}$ & $\%$ & & \\
\hline $\begin{array}{l}\text { Cuidado } \\
\text { Valoración del Ser Humano Unitario. Modalidades de } \\
\text { cuidados en su mayoría no invasivos. Promover la } \\
\text { armonía y bienestar durante la vida. Innovación en } \\
\text { servicio y conocimientos actualizados. }\end{array}$ & 17 & $12 \%$ & 120 & $88 \%$ & 137 & $100 \%$ \\
\hline $\begin{array}{l}\text { Persona } \\
\text { Identifica al paciente solo por los patrones que tiene. } \\
\text { Utiliza el término persona para señalar al ser abierto, } \\
\text { con capacidad de actuar, con sinergia, con el universo. }\end{array}$ & 31 & $23 \%$ & 106 & $77 \%$ & 137 & $100 \%$ \\
\hline $\begin{array}{l}\text { Salud } \\
\text { Es el bienestar, la autorrealización, la actualización y la } \\
\text { realización del potencial de la persona. }\end{array}$ & 11 & $8 \%$ & 126 & $92 \%$ & 137 & $100 \%$ \\
\hline $\begin{array}{l}\text { Entorno } \\
\text { Un sistema abierto y un campo de energía continuo } \\
\text { que no cesa de renovarse. }\end{array}$ & 18 & $13 \%$ & 119 & $87 \%$ & 137 & $100 \%$ \\
\hline
\end{tabular}

En la tabla 2, se observa que los profesionales de enfermería utilizan el $12 \%$ en cuidado, $23 \%$ en el $88 \%$ en cuidado, $77 \%$ en persona, $92 \%$ en salud y $87 \%$ en entorno.

persona, $8 \%$ en salud, $13 \%$ en entorno; pero no utilizan

Tabla 3

Distribución porcentual de la utilización de los Modelos y Teorías de Enfermería de la Escuela del Caring por las enfermeras del Hospital María Auxiliadora. Lima-2011.

\begin{tabular}{|c|c|c|c|c|c|c|}
\hline \multirow{3}{*}{ Dimensiones } & \multicolumn{6}{|c|}{ Escuela Caring } \\
\hline & \multicolumn{2}{|c|}{ Utilizan } & \multicolumn{2}{|c|}{ No utilizan } & \multirow[t]{2}{*}{ Total } & \multirow[t]{2}{*}{$\%$} \\
\hline & $\mathrm{N}$ & $\%$ & $\mathrm{~N}$ & $\%$ & & \\
\hline $\begin{array}{l}\text { Cuidado: } \\
\text { Proceso intersubjetivo de humano a humano que } \\
\text { requiere compromiso. }\end{array}$ & 5 & $4 \%$ & 132 & $96 \%$ & 137 & $100 \%$ \\
\hline $\begin{array}{l}\text { Persona: } \\
\text { Forma viviente que comprende cuerpo, alma y } \\
\text { espíritu. }\end{array}$ & 75 & $55 \%$ & 62 & $45 \%$ & 137 & $100 \%$ \\
\hline $\begin{array}{l}\text { Salud: } \\
\text { Es la armonía entre el cuerpo, el alma y el espíritu. }\end{array}$ & 95 & $69 \%$ & 42 & $31 \%$ & 137 & $100 \%$ \\
\hline $\begin{array}{l}\text { Entorno: } \\
\text { Aspectos contextuales en los que se encuentran los } \\
\text { individuos y los grupos culturales. }\end{array}$ & 34 & $25 \%$ & 103 & $75 \%$ & 137 & $100 \%$ \\
\hline
\end{tabular}

En la tabla 3, se muestra las concepciones de cuidado, persona, salud y entorno, los profesionales de enfermería; utilizan 55\% en persona y $69 \%$ en salud; pero no utilizan el $96 \%$ en cuidado, $45 \%$ en persona y $75 \%$ en entorno. 


\section{DISCUSIÓN}

La Enfermería tiene su origen en las necesidades de cuidado que, desde el inicio de la vida, ha requerido el ser humano para mantener y preservar la vida. El cuidado debe estar dirigido a mantener la funcionalidad del hombre mediante la satisfacción de necesidades de alimentación, protección, vestido y vivienda. La actividad de cuidar ha sido atribuida a las personas que se dedicaron a la Enfermería desde los albores de las civilizaciones (Colliére 1993). Estos parámetros son descritos a través de las teorías y modelos de Enfermería los cuales surgen desde la iniciativa de Florencia Nightingale. Existen pocos estudios en relación a modelos y teorías de Enfermería en nuestro medio. La Enfermería como disciplina depende de la existencia de un conocimiento teórico para todas las áreas (Marriner 2003 \& Potter 2002).

El uso de un modelo conceptual o teoría de Enfermería, en la atención, garantiza que todas las enfermeras compartan igual concepción del metaparadigma o igual lenguaje en la orientación de los cuidados (Campo, Oriach, Viladot 1999). La principal diferencia entre teoría y modelo conceptual es el nivel de abstracción. Un modelo conceptual es un sistema abstracto de conceptos relacionados entre sí. Una teoría está basada en un modelo conceptual, pero está más limitada en el ámbito, contiene más conceptos concretos con definiciones y explicaciones detalladas de las premisas o hipótesis, uniéndolas a todas (Kozier 1993).

Por lo general, teorías, modelos y sistemas conceptuales poseen una estructura idéntica, todos ellos compuestos por enunciados de relación que pretenden describir o explicar los fenómenos y los sistemas (Marriner \& Raile, 1999). Los modelos y teorías nos ayudarán a "adquirir conocimientos que permiten perfeccionar las prácticas cotidianas mediante la descripción, explicación, predicción y control de los fenómenos" (Marriner 2003).

Los modelos y teorías de Enfermería se dividen en metaparadigmas, filosofías, modelos conceptuales, grandes teorías, teorías y teorías de nivel medio. El metaparadigma es el nivel de conocimientos más abstracto de todos. Describe los principales conceptos que hacen referencia al tema principal, así como a la finalidad de una disciplina. Los principales conceptos del metaparadigma enfermero son el de persona, entorno, salud y enfermería. Tal como lo reafirma Fawcet (1984).

Las enfermeras coincidimos en identificar unos elementos característicos y definitorios de nuestra práctica. Estos elementos componen lo que denominamos marco conceptual, marco referencial o metaparadigma enfermero y estos elementos se les nombra utilizando las etiquetas de: persona, salud, entorno, enfermería y cuidado.

Según el desarrollo histórico de la Enfermería, Kérouac (1996) distingue tres paradigmas: Categorización (1850-1950): Los fenómenos son divisibles. Las relaciones que se establecen son lineales y causales. La enfermera suple las incapacidades, es la que tiene el conocimiento.

Integración (1950-1975): La persona es el eje central de la disciplina. La relación es circular e interaccional. La enfermera es consejera, ayuda a escoger los comportamientos de salud.

Transformación (1975-actualidad): El fenómeno se describe como único, complejo y global. La enfermera es partícipe junto a la persona de los cuidados. Existe un gran número de enfermeras asistenciales que no encuentran aplicabilidad de los modelos teóricos en el trabajo diario (Medina 2005) y se preocupan por su habilidad técnica y el dominio de los procedimientos, sin echar de menos la aplicación de una metodología para planificar los cuidados (López 2000). Probablemente por la alta tecnificación, los profesionales se encuentran en el paradigma de la categorización.

Si bien el paradigma de transformación se puede situar temporalmente en la mitad de los años 70, se puede encontrar su impronta en los modelos y teorías propuestos por R.R. Parse, M. Newman, M. Rogers y J. Watson. Dichas teorías y modelos se ubican en las escuelas del ser humano y el caring.

De acuerdo a los resultados, del presente estudio de investigación, se evidencia que existe un bajo porcentaje de enfermeras que utilizan las concepciones de los modelos y teorías de las escuelas del ser humano unitario y el caring. Siendo la dimensión más utilizada salud y persona con $39 \%$ y $34 \%$ respectivamente, pero la dimensión cuidado y entorno es inferior al $20 \%$.

Los conceptos del metaparadigma enfermero tienen, en los modelos y teorías característicos del paradigma de transformación, un significado que según las dimensiones se dice que la persona es considerada como un ser único, entendiendo que sus múltiples dimensiones forman una unidad. Este ser entero $\mathrm{y}$ único es indisociable de su universo y es mayor y diferente que la suma de sus partes. En relación a la dimensión salud es una experiencia que engloba la unidad ser humano-entorno, vale decir involucra un solo contexto. Es evidente que las enfermeras en 
su minoría puedan tener estas concepciones de las dimensiones persona y salud.

Por otro lado, la dimensión entorno es ilimitada, es el conjunto del universo; la dimensión cuidado se orienta hacia la consecución del bienestar de la persona tal y como ella lo define. De este modo cuidar a la persona implica reconocer sus valores culturales, sus creencias y sus convicciones. Estas concepciones de entorno y cuidado son utilizadas por las enfermeras en un número más reducido.

Por tanto, se puede deducir que las enfermeras, en su mayoría no utilizan las concepciones de las teorías de la escuela del ser humano y el caring, según las dimensiones persona, salud, entorno y cuidado.

Por último, se puede analizar los modelos y teorías en lo que Kerouác (1996), denomina escuelas. El significado de las escuelas es homologable al que tiene el término tendencia. La clasificación de los modelos y teorías en escuelas supone un nivel de concreción, mucho mayor al que permite el paradigma. Es decir, al analizar los modelos y teorías se puede establecer un primer nivel de relación entre ellos que viene marcado, precisamente, por su pertenencia a un paradigma.

A continuación, y una vez situados en cada paradigma, se puede buscar un segundo nivel de relación, de manera que dentro de un mismo paradigma se puede encontrar diversas orientaciones, lo que hace que los modelos, que mantienen relación por su pertenencia a dicho paradigma, puedan ser agrupados en varias categorías, en función de la orientación que los relaciona. De esta manera, un modelo o teoría se relacionará con otro, porque ambos comparten un mismo paradigma y con otro, porque además de compartir paradigma, comparte una misma orientación dentro de él.

Los modelos y teorías según S. Kérouac (1996) aparecen agrupados en seis escuelas como son: escuela de necesidades, escuela de interacción, escuela de los efectos deseables, escuela de la promoción de salud, escuela del ser humano unitario y escuela del caring. Los resultados de la utilización de los Modelos y Teorías de Enfermería de la Escuela del Ser Humano Unitario por las enfermeras fue inferior al $25 \%$, es decir, que la cuarta parte al menos tiene nociones de las tendencias de esta escuela, tanto en las dimensiones persona, entorno, cuidado y salud.

En la Escuela del Ser Humano Unitario, la característica fundamental de los modelos y teorías que pertenecen a esta escuela es el interés que demuestran por hacer patente quién es y cómo es el receptor de los cuidados de Enfermería. Dentro de esta escuela se situarían M. Rogers, M. Newman y R.R. Parse mencionado por Kerovac (1996). En la dimensión persona, se identifica al paciente solo por los patrones que tiene, además utiliza el término persona para señalar al ser abierto con capacidad de actuar con sinergia con el universo. En la dimensión entorno, considera un sistema abierto y un campo de energía continuo que no cesa de renovarse.

En la dimensión Cuidado, la enfermera tiene la concepción de Ser Humano Unitario durante la valoración del usuario, la modalidad de cuidados en su mayoría no deben ser invasivos, además de promover la armonía y bienestar durante la vida, teniendo en consideración los cambios mediante la innovación en servicio y conocimientos actualizados.

En la dimensión salud, es el bienestar, la autorrealización, la actualización y la realización del potencial de la persona considera un sistema abierto $y$ un campo de energía.

Al respecto, Martha E. Rogers afirma el modelo de seres humanos unitarios es un campo de energía dinámico que se integra con los campos de energía del entorno. "En el cual se ve al hombre como una totalidad unificada que posee su propia integridad y que manifiesta unas características que son más que y diferentes de la suma de sus partes". Considera a la Enfermería como una profesión que se aprende como una ciencia tanto como un arte, es una ciencia humanística dedicada a la compasión, a mantener y promover la salud, prevenir la enfermedad y asistir y rehabilitar al enfermo y al discapacitado, establece una interacción entre el entorno y el hombre, la integridad de los seres humanos y la búsqueda de un potencial máximo de salud (Kerouác 1996).

El objetivo del modelo, de Martha Rogers, es procurar y promover una interacción armónica entre el hombre y su entorno. Así, las enfermeras que sigan este modelo deben fortalecer la conciencia e integridad de los seres humanos, y dirigir o redirigir los patrones de interacción existentes entre el hombre y su entorno, para conseguir el máximo potencial de salud. Se puede deducir que, en su mayoría, las enfermeras carecen de la concepción de la Escuela del Ser Humano Unitario en las dimensiones persona, entorno, cuidado y salud,

Sin embargo, los resultados en la utilización de los Modelos y Teorías de Enfermería de la Escuela del Caring por las enfermeras, alcanzaron cifras mayores al $50 \%$ en las dimensiones persona y salud, y menos del $25 \%$ en las dimensiones entorno y cuidado. 
En la Escuela del Caring, la característica fundamental de los modelos y teorías que pertenecen a esta escuela es el interés que demuestran por la cultura y lo que se denomina caring. Este nuevo concepto implica un cuidado centrado en la cultura, los valores y las creencias de las personas. Dentro de esta escuela se situarían M. Leininger y J. Watson mencionados por Kerouác (1996).

Se está enfatizando actualmente las concepciones de la Escuela Caring, así se tiene en la dimensión salud, la enfermera considera que tener salud es armonizar el cuerpo, el alma y el espíritu, por otro lado, tiene en cuenta a la persona como una forma viviente que tiene cuerpo alma y espíritu. En relación al entorno, es bajo el porcentaje que concibe al usuario y grupos culturales dentro de un contexto. Un número reducido también, el 5\%, utiliza la concepción de los procesos intersubjetivos de humano a humano, esto implica que se carece de esa comunicación, trato afectivo entre los seres humanos. Se puede deducir que en su mayoría las enfermeras carecen de la concepción de la Escuela del Caring Unitario en las dimensiones salud, persona, entorno y cuidado.

Los resultados sugieren, entre las conclusiones, que el uso de las teorías de Enfermería no depende de la organización, ni del número de enfermeras y de pacientes, ni de dónde provienen las teorías, solo dependerá de la percepción que tengan las enfermeras de la necesidad de uso de un marco teórico que fundamente y guíe la práctica, y de una concepción de la teoría en el conocimiento para su aplicabilidad en la práctica.

Es evidente que cada vez más se percibe la falta de aplicabilidad o uso de tales modelos y teorías de Enfermería, a pesar de la insistencia de incorporarlas en la formación del profesional de Enfermería (Marriner, 2003).

Sin embargo, es necesario promover la investigación, en relación a la utilidad de las teorías de Enfermería como fundamento del conocimiento de este grupo profesional, con la finalidad de mejorar la calidad del servicio y el desarrollo de la profesión. Otro estudio presentado por Santos, López \& Varez (2007), sobre perspectiva en cuanto a modelos y teorías de enfermería en el ámbito de nefrología, algunos resultados muestran que un $22,2 \%$ afirma seguir algún modelo en la actividad diaria (Henderson), un $66,7 \%$ contesta que no; consideran, entre algunas conclusiones, que se evidencia una gran variabilidad en cuanto al posicionamiento teórico de los profesionales.
A menudo, los modelos y teorías de Enfermería se relegan de la práctica enfermera porque son complejos y no se conocen en profundidad; sin embargo, es una gran responsabilidad de las enfermeras que la aplicación de las teorías y modelos, en el cuidado, tenga fundamentos científicos sólidos, permitiendo así el avance de la ciencia enfermera. Existe un gran número de enfermeras asistenciales que no encuentran aplicabilidad de los modelos teóricos en el trabajo diario (Medina 2005).

Por otro lado, el paradigma de transformación ubica a las escuelas del ser humano y el Caring, sin embargo, las concepciones de estas teorías en su dimensión persona, salud, entorno y el Caring, son poco utilizadas por el profesional de Enfermería.

La Escuela del Ser Humano Unitario conceptualiza a la Enfermería como ciencia humanística que procura y promueve una interacción armónica entre el hombre y su entorno, integrando a los seres humanos y buscando un potencial máximo de salud, esta concepción es utilizada en su minoría por el profesional de Enfermería, tanto en las dimensiones persona, entorno, cuidado y salud.

En la Escuela del Caring se conceptualiza un cuidado centrado en la cultura, los valores y las creencias de las personas, esta concepción es utilizada por el profesional de Enfermería, en su mayoría, en las dimensiones persona y salud y, en su minoría, en las dimensiones entorno y cuidado.

El profesional de Enfermería presenta áreas críticas en la utilización de los modelos y teorías de las escuelas del ser humano unitario, tanto en la conceptualización de persona, entorno, salud y enfermería, y en la escuela del caring, en la conceptualización entorno y cuidado.

Declaración de financiamiento y de conflicto de intereses:

El estudio fue financiado por los autores, quienes declaran no tener algún tipo de conflicto de interés en la investigación realizada.

\section{Correspondencia:}

\section{Nancy Huamán Salazar}

EP Enfermería, Facultad de Medicina. Universidad Nacional Mayor de San Marcos. Av. Grau No 755. Lima. Perú.

e-mail: nhuamans@hotmail.com 


\section{REFERENCIAS BIBLIOGRÁFICAS}

Armas, E. (2010). Factores influyentes en la aplicación de modelos y teorías de enfermería para el cuidado del paciente. Revista Cientifica de Enfermería. 2(1), 16-21.

Campo, M., Oriach, M., Viladot, A., Espinalt, A. \& Fernández, C. (1999). Servicio de cuidados enfermeros según modelo conceptual de Virginia Henderson. Implantación, resultados y costes. Metas de Enfermería; 2(13), 8-14.

Durán de Villalobos, M. (2002). Marco epistemológico de la enfermería. Revista Aquichan. 2(2)

Fawcet, J. (1984). Analysis and evaluation of conceptuals models. Philadelphia: Davis.

Hernández, C. (1999). Fundamentos de la enfermería. Mc Graw Hill: España.

Kérouac, S., Pepin, J., Ducharme, F., Duquette, A. \& Major, F. (1996). El pensamiento enfermero. Masson: Barcelona - España.

Kozier, B., Erb, G. \& Olivieri, R. (1993). Enfermería fundamental. Editorial McGraw-Hill. Interamericana: Madrid: Vol. I.4 ${ }^{\mathrm{a}}$ edición.

López J. (2000). Propuestas para el desarrollo profesional (I). Enfermería Clínica, 10(3),114-118.

Marriner T. \& Raile, A. (2011). Modelos y teorías en enfermería. Barcelona: Elsevier Mosby.
Marriner, A. (2003). Modelos y teorías de enfermería. Barcelona: Editorial. Elsevier Science.

Medina, J. (2005). ¿Teoría? ¿Práctica? El difícil equilibrio académico y profesional en la España "pre Europea" (II). Revista Rol Enfermería, 28(4), 246-248.

Potter, P. \& Perry, A. (2002). Fundamentos de enfermería. España- Madrid: Hartcourt-Mosby.

Santos, S., López, M. \& Varez, S. (2007). Aplicabilidad de modelos y teorías de enfermería en la realidad asistencial. XI Encuentro Internacional de Investigación en Enfermería. ISBN: 13978-84-690-90251. Edita: Investén- ISC III. España. p. 50-52.

Varez, S., Parra, M., Santos, S. \& Sabater, A. (2008). Perspectiva sobre modelos y teorías de enfermería en el ámbito de nefrología. Revista de la Sociedad Española de Enfermería Nefrológica, 11(3), 18-22.

Vásquez, T. \& Martha, L. (2007). Desarrollo conceptual en enfermería: Evolución, tendencias y desafíos. Revista Avances, 25(1), 73-80.

Recibido: $15 / 11 / 2014$ Aceptado: 15/03/2015 